\title{
PROGRESS OF THE LASER ELECTRON ACCELERATOR PROJECT AT STANFORD UNIVERSITY*
}

\author{
R.L. Byer, T. Plettner†, Ginzton Laboratories, Stanford University \\ C. Barnes, E. Colby, B. Cowan, R.H. Siemann, J.E. Spencer, SLAC
}

\begin{abstract}
Our objective is to demonstrate, via a proof-ofprinciple experiment, particle acceleration by crossed laser beams in a single dielectric accelerator cell in vacuum. We expect to observe a laser-induced energy modulation of $15 \mathrm{keV}$ on an electron beam with an initial energy of $32 \mathrm{MeV}$.
\end{abstract}

\section{INTRODUCTION}

Crossed laser beam acceleration shares several common aspects with RF linear acceleration: the acceleration occurs in vacuum and both schemes rely on a structure that provides a longitudinal electric field responsible for the acceleration. The design of the structure is chosen to prevent a change of sign of the phase of the longitudinal field. The damage threshold from the electric field of the structure is the limiting factor for the acceleration gradient for both schemes. One important difference between the two schemes is the choice of material for the accelerator structure. While RF structures are typically constructed from conductors, laser structures can be fabricated from dielectric materials that are capable of withstanding higher electric fields before breaking down.

With ultra-short, near-infrared laser pulses, dielectric materials show a very large damage threshold [1],[2]. For laser pulses of $100 \mathrm{fsec}$ they can tolerate peak electric fields of $10 \mathrm{GV} / \mathrm{m}$, allowing for a structure capable of supporting an average energy gradient of 1 $\mathrm{GeV} / \mathrm{m}$. In addition, the structure design is chosen such that the electron beam never traverses any material, preventing beam loss and emittance growth. This makes crossed laser beam acceleration a very promising scheme for reaching the high-gradient of the next generation particle accelerator. The mechanism of crossed laser beam acceleration has been described by several authors in the past [3],[4],[5] and accelerator structure designs have been proposed [6],[7] but to our knowledge no experimental verification for this acceleration scheme has been carried out so far.

We have been working on a proof-of-principle experiment at the SCA-FEL facility at Stanford University. Table 1 summarizes the laser and electron beam characteristics at this facility. The overall experimental setup is described in publications [8],[9].

*Supported by DOE contract DE-FG03-97ER41043

$\dagger$ Email: tplettne@ stanford.edu
Table 1:SCA-FEL beam parameters

\begin{tabular}{|c|l|l|}
\hline \multirow{3}{*}{$\begin{array}{l}\text { laser } \\
\text { beam }\end{array}$} & Wavelength & $800 \mathrm{~nm}$ \\
\cline { 2 - 3 } & Typical pulse width & $2 \mathrm{psec}$ \\
\cline { 2 - 3 } & $\begin{array}{l}\text { Maximum energy per } \\
\text { pulse }\end{array}$ & $0.15 \mathrm{~mJ}$ \\
\cline { 2 - 3 } & FWHM spot size & $160 \mu \mathrm{m}$ \\
\hline \multirow{3}{*}{$\begin{array}{c}\text { electron } \\
\text { beam }\end{array}$} & Energy & $32 \mathrm{MeV}$ \\
\cline { 2 - 3 } & Expected bunch length & $1-2 \mathrm{psec}$ \\
\cline { 2 - 3 } & Electrons per bunch & $10^{7}$ \\
\hline
\end{tabular}

Here we report on the recent progress and describe the modifications carried out during the last year. Figure 1 shows the current interaction region layout. The electron beam interacts with the laser beam pair over a distance of $1.5 \mathrm{~mm}$ inside a dielectric accelerator cell. A high-resolution energy spectrometer downstream records the energy spectrum of the electron beam.

\section{RECENT PROGRESS}

\subsection{Spatial and temporal overlap diagnostics}

In the past we lacked a precise monitor for spatial overlap between the electron and the laser beam as well as a precise absolute timing diagnostic. A major redesign of the accelerator cell was performed in order to accommodate these diagnostics. Figure 1 illustrates the the modified accelerator cell and its arrangement with the spatial and temporal diagnostics.

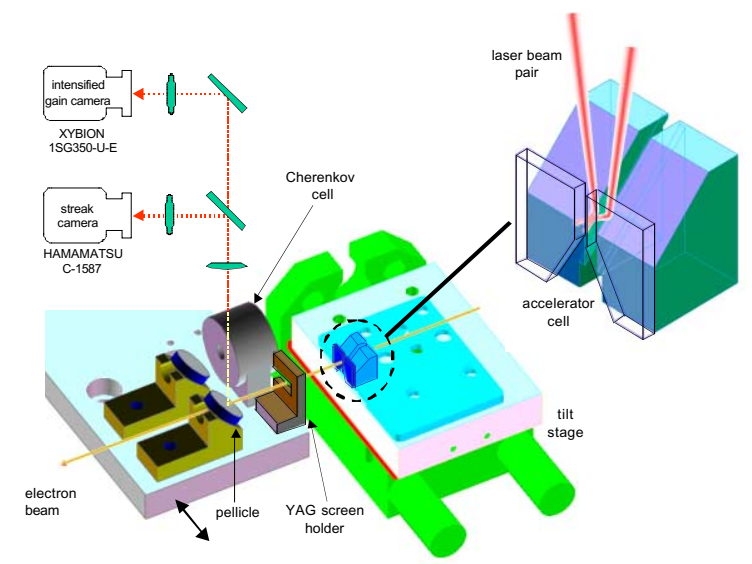

Figure 1: The modified accelerator cell and the spatial and timing monitors described in the text 
To monitor absolute timing overlap a cell containing aerogel for Cherenkov radiation can be moved immediately downstream of the accelerator cell such that both the electron beam and the laser beam traverse the cell and reach a Hamamatsu C-1587 streak camera through the same optical path. The time difference between the laser and the Cherenkov radiation can be monitored with a resolution of $\sim 20$ psec.

The spatial overlap between the laser and the electron beam is monitored with a XYBION ISG350 intensified, gated camera, which observes a YAG fluorescent screen that can also be placed immediately behind the accelerator cell. The Cherenkov cell and the YAG screen are mounted on a common platform that can be translated laterally to select the diagnostic to be located downstream of the accelerator cell. Both diagnostics can be moved out of the electron beam trajectory.

\subsection{Tungsten electron beam collimator}

The large position jitter of the electron beam at the accelerator cell location forced us to insert a tungsten mask with a $100 \mu \mathrm{m}$ slit aligned to the laser beam at the exit of the accelerator cell to remove electrons that would not experience the laser fields. The nearly $\pi / 2$ betatron phase advance between accelerator cell and dispersive plane would otherwise fold these "spectator" electrons on top of the accelerated electrons.

\subsection{Laser optical phase monitor}

In order to produce an accelerating field component a phase shift of $\pi$ between the split laser beams is required. The optical phase between the lasers is controlled with a piezo crystal-mounted retroreflector and monitored by observing the laser field that exits the accelerator cell. Minimum brightness of this field corresponds to the laser beams being out of phase by $\pi$ . This monitor also allows us to confirm that the relative phase between the laser beams has no significant shortterm or long-term jitter.

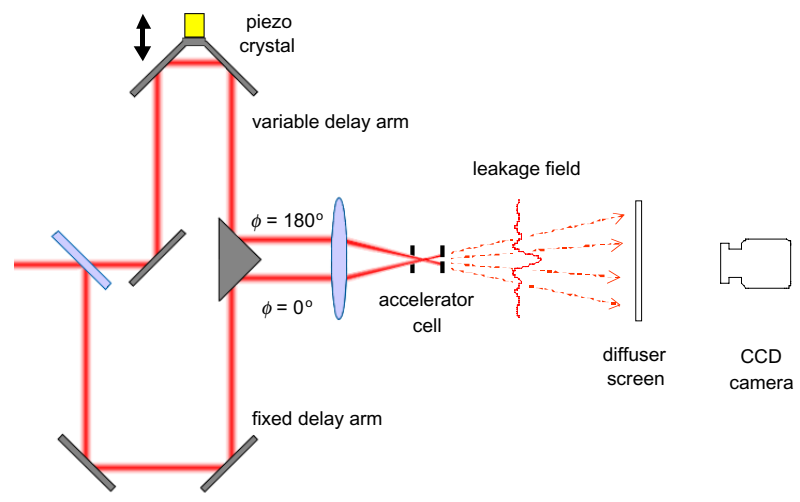

Figure 2: Laser-optical phase control and monitoring

\subsection{Accelerator cell damage monitor}

The selected laser power depends on the damage threshold of the accelerator cell, which varies from sample to sample. When damaged, the dielectric surface and interference pattern deteriorate, and the acceleration field is destroyed. A device that detects damage of the cell has to be present in the experiment. An effective scheme for this purpose is to monitor the laser beam that is reflected back from the accelerator cell. A diagram of the damage monitor is depicted in Figure 3.
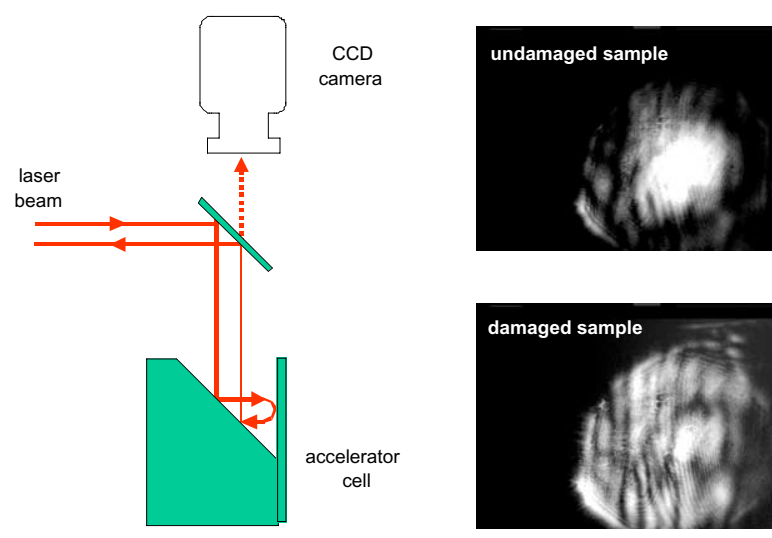

Figure 3: The cell damage monitor

Preliminary tests have shown varying damage threshold values for the multi-layer dielectric coated fused silica accelerator cell under vacuum. The lowest observed damage threshold value corresponded to a peak fluence of $0.27 \mathrm{~J} / \mathrm{cm}^{2}$ at the bright fringes of the interfering laser beam pattern. The published fluence value at damage threshold for fused silica in air is $2 \mathrm{~J} / \mathrm{cm}^{2}$.

\section{SELECTION OF PARAMETERS}

Evidence for laser-acceleration will manifest itself as a broadening of the energy spectrum. A summary of the parameters that optimize the broadening of the energy spectrum for a $32 \mathrm{MeV}$ beam is presented in Table 2 . The parameter choice is discussed in reference [9].

Table 2: Selected experimental parameters

\begin{tabular}{|l|l|}
\hline $\begin{array}{l}\text { Laser : e-beam interaction } \\
\text { length }\end{array}$ & $1500 \mu \mathrm{m}$ \\
\hline Laser pair crossing angle & $32 \mathrm{mrad}$ \\
\hline Accelerator slit width & $<10 \mu \mathrm{m}$ \\
\hline laser pulse duration & $2 \mathrm{psec}$ \\
\hline
\end{tabular}

The spot size of the interfering laser beams at the accelerator cell is $160 \mu \mathrm{m}$ FWHM, corresponding to a Rayleigh range of $\sim 7 \mathrm{~cm}$. The slippage distance is 1.5 $\mathrm{mm}$. At a fluence of ${ }_{-} \mathrm{J} / \mathrm{cm}^{2}$ the average gradient is 10 $\mathrm{MV} / \mathrm{m}$, and an energy gain of $15 \mathrm{keV}$ is expected. 


\section{OBSERVED ENERGY SPECTRUM}

The SCA beam shows shot-to-shot jitter as well as various types of long term drifts. Large fluctuations in the intensity and energy of the profiles forced us to perform some numerical processing to the individual energy profiles in order to be able to compare them. The peak of each profile was normalized to unity and was centered on the energy scale. Figure 4 shows the processed energy profiles of 1000 successive shots.

Figure 4: Energy spectrum of 1000 successive shots

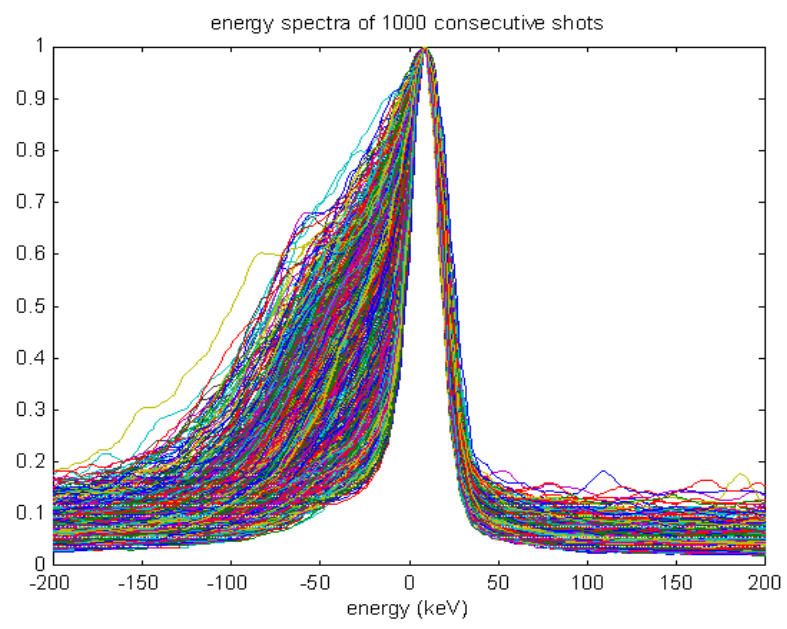

As observed in Figure 4, in addition to the energy and intensity fluctuations an asymmetry in the profile is clearly visible: a long low-energy tail with large shotto-shot fluctuations and a sharp high energy edge only a few $\mathrm{keV}$ wide. The asymmetric shape and overall width of the profile do not depend on the slit width of the accelerator cell, indicating that wakefield effects are negligible. With a standard deviation of $7 \mathrm{keV}$ the high energy side of the energy profiles is the clear choice for observing a laser induced energy broadening.

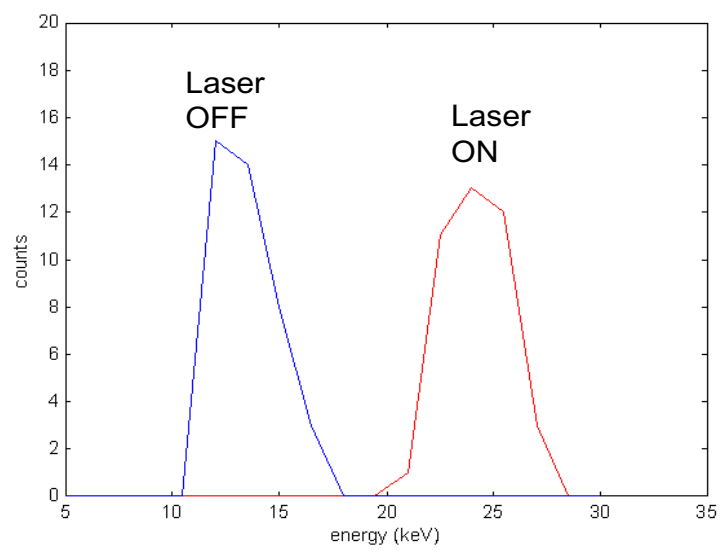

Figure 5: Expected laser induced energy broadening

The difficulty of the experiment becomes evident from figure 5, which shows a histogram of the fitted widths on the high-energy side of the distribution with
("Laser on") and without ("Laser off") laser acceleration. At a laser power level near the damage threshold of the accelerator cell an energy modulation of $15 \mathrm{keV}$ is expected and an overall system resolution of $<0.05 \%$ is required..

\section{FUTURE DIRECTIONS}

With the small energy modulation induced by the laser fields, it is important to reduce the number of "spectator" electrons present in the measured energy profile. We hope to implement bunch length monitoring to permit direct monitoring of the pulse shape to permit maximum overlap of the electron and photons. By modulating the crossed laser beam relative phase and using synchronous detection techniques, we expect to further enhance our detection sensitivity.

Beam transmission through $5 \mu \mathrm{m}$ slits has proven to be straightforward, allowing us to move from an adjustable gap cell to a fixed gap cell. This simplification will permit lithographic techniques to be used to produce highly precise, simple accelerator cells, and to try several different cells in a single experiment and to relax concerns about laser damage.

\section{ACKNOWLEDGEMENTS}

The authors thank $\mathrm{H}$. Wiedemann and his group for their assistance with bunch length measurements, and M. Hennessy for his extensive and prompt help with the vacuum system. We are also grateful for the assistance of the staff of Hansen Laboratories in operating the accelerator. Finally, we thank Prof. S. Harris for lending his streak camera to us, and Dick Sturm for supplying several robust XYBION image intensifiers.

\section{REFERENCES}

[1] G. Mourou, et al, "Laser induced breakdown by impact ionization in $\mathrm{SiO} 2$ with pulse widths from $7 \mathrm{~ns}$ to $150 \mathrm{fs}$," Appl. Phys. Lett, June, 1994.

[2] B.C. Stuart, M.D. Feit, A.M. Rubenick, B.W. Shore, and M.D. Perry, Phys. Rev. Lett. 74 (12) 2248 (1995).

[3] J.A. Edinghofer and R.H. Pantell, "Energy exchange between free electrons and light in vacuum", J. Appl. Phys. 50(10) 6120 (1979).

[4] C.M. Haarland, "Laser Electron Acceleration in Vacuum", Opt. Comm. 114, 280 (1995).

[5] P. Sprangle, E. Esarey, J. Krall, A. Ting, Opt. Comm. 124, 69 (1996).

[6] Y.C. Huang, D. Zheng, W.M. Tulloch, R.L. Byer, "Proposed structure for a crossed-laser beam, GeV per meter gradient, vacuum electron linear accelerator", Appl. Phys. Lett. 68 (6) 753 (1996).

[7] Y.C. Huang, R.L. Byer, "A proposed high-gradient laserdriven electron accelerator using crossed cylindrical focusing" Appl. Phys. Lett. 69 (15) 2175 (1996).

[8] T. Plettner et al, Conference paper, AAC (1998).

[9] T. Plettner et al, Conference paper, PAC (1999). 